\title{
AVALIAÇÃO DA SEGURANÇA E OCORRÊNCIA DE DEFEITOS NA OPERAÇÃO DE CORTE SEMIMECANIZADO DE FLORESTAS DE EUCALIPTO ${ }^{1}$
}

\author{
Reginaldo Sergio Pereira², Patrick Bruno Ruas Guimarães ${ }^{3}$, Cláudio Henrique Soares Del Menezzi², Ailton
} Teixeira do Vale ${ }^{2}$ e Renato César Gonçalves Robert ${ }^{4}$

\begin{abstract}
RESUMO - Avaliou-se a operação de corte semimecanizado nos aspectos de segurança no trabalho de operadores de motosserra e na qualidade da madeira derrubada, visando validar as recomendações técnicas da literatura especializada. O estudo foi realizado em povoamentos de Eucalyptus grandis com idade de 9 anos plantados em espaçamento 3 x 2 m, na Estação Experimental Fazenda Água Limpa (FAL), pertencente à Universidade de Brasília. Foram estabelecidos procedimentos relativos à técnica de derrubada de árvores. O tratamentotestemunha foi executado segundo as recomendações técnicas da literatura especializada. Os demais tratamentos variaram em relação à profundidade e ângulo de execução da boca de corte e, também, à altura de execução do corte de queda das árvores. Avaliaram-se a segurança da operação, ocorrência de rachaduras de topo, formação de espetos e volume de madeira remanescente na cepa, sendo os tratamentos comparados pelo teste de Dunnett. Pelos resultados, o tratamento-testemunha mostrou-se a melhor alternativa técnica para a derrubada de árvores de eucalipto com motosserra, nos aspectos de segurança e qualidade da madeira.
\end{abstract}

Palavras-chave: Motosserra. Segurança. Defeitos.

\section{SAFETY AND OCCURRENCE OF DEFECTS IN THE SEMI-MECHANIZED CLEAR-CUTTING OPERATION OF EUCALYPT FORESTS}

\begin{abstract}
The semi-mechanized clear-cutting operations were evaluated regarding to chainsaw operator safety and to the quality of the felled log aiming at validating the thecnical recommendations from literature. This study was conducted in an Eucalyptus grandis plantation (spacing of $3 m \times 2 m$ ) established on Fazenda Água Limpa (FAL), which is an experimental area of the University of Brasilia. Ten treatments related to the tree falling techniques were proposed, whereas the control treatment was carried out according to the recommended procedures for fell trees according to the literature. According to the proposed treatments, the following variables were evaluated: deep and angle of the wedge cut, height of wedge cut to fell the tree. For each treatment the (a) operation safety, (b) log end-splitting, (c) fork formation and (d) remained volume of the tree were analyzed. Control and proposed treatments were compared by using the Dunnett test. According to the results, the control treatment showed the best result and it should be recommended to fell tree by using chainsaw.
\end{abstract}

Keywords: Chainsaw. Security. Defect.

\section{INTRODUÇÃO}

No Brasil, apesar da intensificação da mecanização da colheita florestal a partir da década de 1990, com a entrada no setor de máquinas com tecnologias avançadas, grande parte do corte de florestas nativas e plantadas para os mais diversos usos tem sido realizado pelo método semimecanizado, ou seja, com a utilização da motosserra.

Uma das características do corte florestal semimecanizado é ser um trabalho com alto risco de

\footnotetext{
${ }^{1}$ Recebido em 25.04.2011 e aceito para publicação em 19.04.2012.

${ }^{2}$ Universidade de Brasília, UNB, Brasil. E-mail <reginaldosp@unb.br>, <cmenezzi@ unb.br>e < ailton.vale@gamil.com>.

${ }^{3}$ Universidade de Brasília, UNB, Brasil. E-mail: <patrickruas@gmail.com>.

${ }^{4}$ Universidade Federal do Paraná, UFPR, Brasil. E-mail: <renatorobert@ufpr.br>.
} 
acidentes. Os trabalhadores atuam expostos às condições climáticas, em diferentes tipos de terreno e de florestas e povoamentos, sujeitos ainda a acidentes com animais peçonhentos (SODERSTROM, 1982). Os problemas relacionados a esse fato dizem respeito, principalmente, à falta de treinamento adequado de operadores associada à alta demanda do mercado por madeira. Esses aspectos podem comprometer a segurança, bem-estar e nível de satisfação do trabalhador florestal, além da qualidade da madeira obtida para algum fim.

Canto et al. (2007) evidenciaram a necessidade de conscientização dos proprietários rurais fomentados e dos trabalhadores sobre o risco de acidentes inerentes à colheita florestal e a importância da utilização de equipamentos de proteção individual, bem como de instrução sobre como trabalhar de forma segura. Jacovine et al. (2005) destacaram que a melhoria da qualidade do processo pode ser alcançada com investimento em treinamento dos trabalhadores florestais.

A colheita e o transporte são as etapas mais importantes do ponto de vista econômico, dada a sua alta participação nos custos finais da madeira posta na indústria (MACHADO; LOPES, 2000). A colheita florestal traz retorno quando da implantação de programas de qualidade, a exemplo de treinamentos (TRINDADE et al., 1991).

A madeira de eucalipto tem sido usada na obtenção de fibra curta para produção de celulose, produção de painéis de madeira, de carvão, postes, moirões etc. Entretanto, segundo Lima et al. (2002), entre as dificuldades que essa espécie pode apresentar estão as rachaduras de topo de toras e de peças serradas que reduzem o aproveitamento da madeira, as quais são devidas às tensões de crescimento, normalmente elevadas nos eucaliptos.

Vários estudos têm sido realizados (SKOLMEN, 1967; AGUIAR; JANKOWSKI, 1986; KUBLER, 1987; ROZAS MELLADO, 1993; LELLES; SILVA, 1997; SEVERO; TOMASELLI, 2000) com madeira de eucalipto objetivando descobrir maneiras de amenizar problemas causados pela forma inadequada de realização do corte florestal.

As tensões de crescimento são desenvolvidas no interior do tronco da árvore viva (JACOBS, 1945). Essas forças se originam na região cambial do tronco, durante a fase de maturação das células, em que elas tendem a se contrair longitudinalmente e se expandir transversalmente (LIMA et al., 2000). Uma consequência importante que pode ser observada nesse fenômeno é o estado de tensão de tração que surge no sentido longitudinal e tensões de compressão no sentido tangencial das toras (WILHELMY; KUBLER, 1973).

Assim, o objetivo deste trabalho foi avaliar a operação de corte semimecanizado em povoamento de eucalipto, nos aspectos referentes à segurança no trabalho e à ocorrência de defeitos na madeira, visando validar as recomendações técnicas da literatura especializada.

\section{MATERIAL E MÉTODO}

\section{1. Área de estudo, povoamento florestal e motosserra}

O trabalho foi realizado na Reserva Ecológica e Experimental da Universidade de Brasília, Fazenda Água Limpa (FAL), localizada a uma altitude de 1.100 $\mathrm{m}$, nas coordenadas $15^{\circ} 56^{\prime}-15^{\circ} 59^{\prime} \mathrm{S}$ e $47^{\circ} 55^{\prime}-47^{\circ} 58^{\prime} \mathrm{WGr}$. O povoamento florestal era composto por árvores da espécie Eucaliptus grandis, com idade de 9 anos, plantadas em espaçamento 3 x 2 m e DAP (diâmetro à altura do peito) variando entre $20 \mathrm{e} 25 \mathrm{~cm}$, em terreno plano. Utilizou-se para o corte semimecanizado uma motosserra da marca Stihl, modelo MS 381, com peso de $6,6 \mathrm{~kg}$, sabre de $40 \mathrm{~cm}$, movida a motor de dois tempos e fabricada no ano de 2010 .

\subsection{Definição dos tratamentos}

Foram derrubadas 30 árvores de eucalipto enquadradas nos seguintes tratamentos:

- Tratamento 1 Técnica correta de corte com motosserra.

- Tratamento 2 Corte retilíneo sem execução da boca de corte.

- Tratamento 3 Boca de corte com profundidade igual a 1/2 do diâmetro da árvore.

- Tratamento 4 Boca de corte com profundidade igual a 1/6 do diâmetro da árvore.

- Tratamento 5 Boca de corte com ângulo de $65^{\circ}$.

- Tratamento 6 Boca de corte com ângulo de $25^{\circ}$.

- Tratamento 7 Corte de queda $10 \mathrm{~cm}$ acima da boca de corte.

- Tratamento 8 Corte de queda na mesma altura da boca de corte. 
- Tratamento 9 Corte de queda $10 \mathrm{~cm}$ abaixo da boca de corte.

- Tratamento 10 Técnica de corte totalmente irregular.

O tratamento de número 1 refere-se à execução da técnica de corte de árvores com motosserra segundo as recomendações da literatura especializada (LOPES et al., 2001; SANT'ANNA, 2008). A técnica consiste em realizar um entalhe direcional constituído de dois cortes, um superior realizado obliquamente em um ângulo predefinido de 45 a $60^{\circ}$ e outro horizontal com profundidade de $1 / 5$ a 1/3 do diâmetro da árvore, medido no ponto de corte. Posteriormente, é realizado o corte de queda, feito do lado contrário à boca de corte, 2 a $5 \mathrm{~cm}$ acima desta, com profundidade igual ou inferior à metade do diâmetro da árvore. A Figura 1 ilustra os procedimentos técnicos descritos para esse tratamento.

Visando avaliar a veracidade dos procedimentos recomendados na literatura para o corte de árvores, realizaram-se também os Tratamentos 2 a 10 . No Tratamento 10, técnica de corte totalmente irregular, a profundidade da boca de corte foi executada na $1 / 2$ do diâmetro da árvore e em ângulo de $25^{\circ}$, e o corte de queda foi executado abaixo $(10 \mathrm{~cm})$ da boca de corte.

\subsection{Parâmetros avaliados}

\section{a) Segurança do operador}

A segurança do operador foi avaliada quanto à dificuldade da operação, medida pelo ciclo operacional de corte, tempo total de execução do corte, quanto ao tempo de queda da árvore, após a execução do corte de abate e, também, quanto ao local de queda da árvore

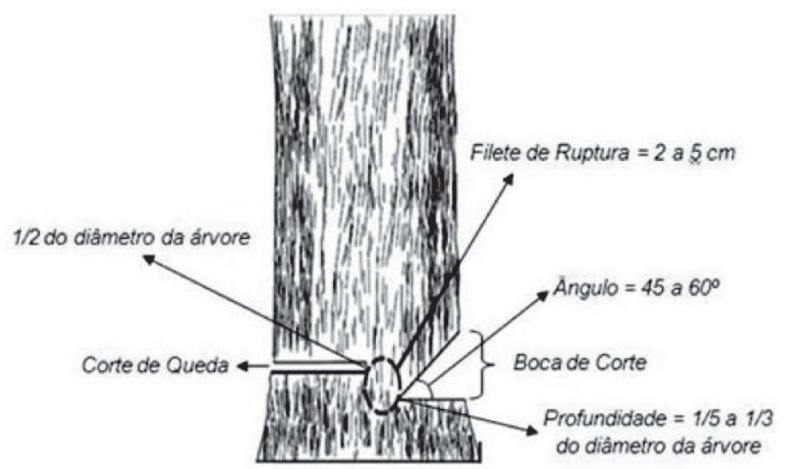

Figura 1 - Técnica correta de derrubada de árvores. Figure 1-Recommended technique to fell trees. previamente planejado. Esses aspectos são fundamentais para a manutenção da integridade física do operador, que após completar o corte da árvore se deslocava para os caminhos de fuga.

Os tempos totais e de queda das árvores, em cada tratamento, foram determinados com o auxílio de um cronômetro digital sexagesimal da marca Technos YP 2151. O tempo total foi contabilizado desde o início do corte até a completa queda da árvore ao solo, enquanto o tempo de queda o foi a partir da finalização do corte de abate até o contato da árvore com o solo. Tanto o tempo de queda quanto o tempo total foram processados em segundos.

O direcionamento de queda da árvore foi avaliado de acordo com a precisão entre o local de queda e o planejamento do seu direcionamento feito antes do início do corte. Avaliou-se a queda das árvores por meio de uma escala, em que foram atribuídos pesos para erros que poderiam ocorrer durante o corte, como se segue:

- Escala 1 - Queda da árvore sem maiores problemas; não houve problema de direcionamento; não houve problema com a motosserra durante o corte; e o tempo de queda foi bom e suficiente para o operador não correr riscos.

- Escala 2 - Não houve problemas de direcionamento; não houve problema com a motosserra durante o corte; a queda da árvore ocorreu em tempo muito rápido; problema de "engaiolamento" da árvore durante a queda.

- Escala 3 -Houve problemas de direcionamento durante a queda da árvore e também com a motosserra durante o corte.

\section{b) Formação de espetos e madeira remanescente nas cepas (altura acima de $10 \mathrm{~cm}$ )}

Após a derrubada das árvores, foi feita uma análise nas cepas remanescentes. Quando presentes, estas foram mensuradas em cada tratamento, sendo medidos (área e altura) os espetos formados e da madeira remanescente nas cepas e, em seguida, estimado o volume $\left(\mathrm{cm}^{3}\right)$ desses defeitos. A área dos espetos foi estimada em percentual, na área total do toco.

\section{c) Rachaduras de topo}

As rachaduras de topo provenientes das tensões de crescimento internas da tora foram medidas, nos

Revista Árvore, Viçosa-MG, v.36, n.3, p.511-518, 2012 
fustes derrubados em cada tratamento, segundo a metodologia proposta por Lima (2002), em que foram feitas medições diretas de comprimento $(\mathrm{cm})$ e largura (cm) das dimensões das rachaduras, após 48 h da derrubada das árvores. O cálculo do índice de rachaduras foi feito de acordo com a equação proposta pelo referido autor, ou seja:

$I R_{P a j}=\frac{\sum_{1}^{n} a_{i} \times C_{i}}{2}$

em que:

$\mathrm{IR}_{\mathrm{Paj}}=$ índice de rachadura ajustado para que o resultado ocorra em cm²;

$\mathrm{a}_{\mathrm{i}}=$ abertura máxima da rachadura $\mathrm{i}(\mathrm{i}=1 \ldots \mathrm{n}), \mathrm{em} \mathrm{cm}$; $\mathrm{C}_{\mathrm{i}}=$ comprimento da rachadura, em $\mathrm{cm}$.

\subsection{ANÁLISE ESTATÍSTICA}

Os resultados de rachadura de topo, formação de espetos e volume da madeira remanescente nas cepas foram analisados estatisticamente pelo teste de Dunnett. Esse teste teve como objetivo a comparação entre cada tratamento e o tratamento-testemunha (T1), procurando-se, através da ANOVA, evidenciar ou não a existência de significância entre os tratamentos. Os dados foram analisados com o auxílio do software SPSS versão 15 .

\section{RESULTADOS}

\subsection{Avaliação da segurança}

Na Figura 2, observa-se que o tempo de queda foi maior nos casos em que houve variação do ângulo da boca de corte (T5 e T6), pois o efeito "alavanca", que é a finalidade do corte direcionado, sofreu interferência, o que proporcionou maior segurança ao motosserrista. Fica evidente nesse ponto que os rendimentos exigidos dos trabalhadores florestais têm que ser associados às questões de segurança. Os tratamentos 9 e 10 foram os que apresentaram tempo de queda menor, decorrente do fato de o corte de derrubada ser realizado $10 \mathrm{~cm}$ abaixo da boca de corte, acarretando risco ao operador da motosserra, que teve menor tempo (aproximadamente $4 \mathrm{seg}$.) para deslocar-se até o caminho de fuga. Os demais tratamentos apresentaram tempos de queda relativamente iguais, variando entre 5,3 e 8,0 seg.

Revista Árvore, Viçosa-MG, v.36, n.3, p.511-518, 2012
Com relação ao tempo total, notou-se variação entre os tratamentos 2 e 3 e os demais. No T2 não foi executada a boca de corte, razão pelo qual foi observado o menor tempo total (22,6 seg.), e no T3 o valor de tempo total obtido (25,0 seg.) se deveu ao tamanho do filete de ruptura, que foi menor do que nos demais tratamentos, em virtude da profundidade da boca de corte. Nos outros tratamentos, o valor médio de tempo total foi de 53,2 seg., com valores máximos e mínimos de 62,1 e 40,0 seg., respectivamente. Os aspectos que provocaram elevado tempo na maioria dos tratamentos foram a dificuldade de realização dos tipos de corte e o "enganchamento" da motosserra.

Ao analisar o resultado alcançado pela escala de queda dos tratamentos (Figura 3), observou-se que, ao realizar cortes totalmente fora dos padrões recomendados, a segurança na operação de derrubada

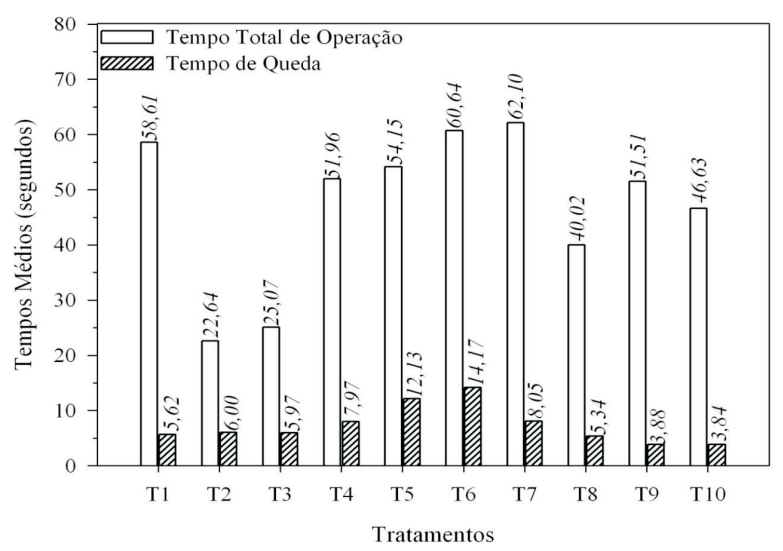

Figura 2 - Média, em segundos, dos tempos de queda da árvore e total.

Figure 2 -Mean values (s) for time for falling and time for finishing the operation.

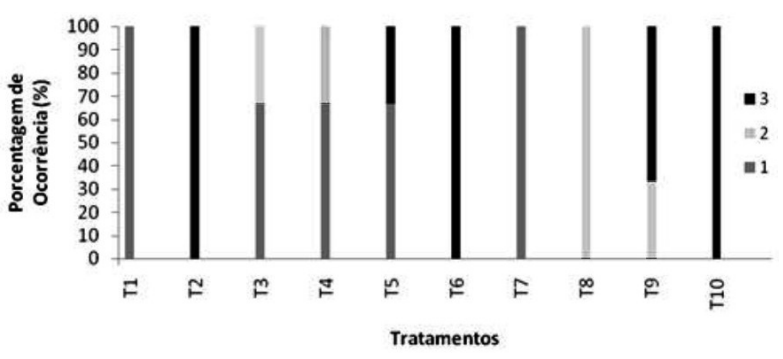

Figura 3 - Escalas de queda dos tratamentos realizados. Figure 3-Fall tree class value of the proposed treatments. 
fica totalmente vulnerável, visto que a árvore não cai no direcionamento esperado pelo operador e ocorrem problemas relacionados tanto com o tempo da derrubada quanto com a motosserra.

Tanto no tratamento em que não é feita a boca de corte (T2) quanto naquele em que o corte é feito de maneira totalmente irregular (T10), a escala de queda de $100 \%$ das árvores é dada como " 3 ”, o que significa que, nesses casos, a segurança do operador e da operação foi afetada como um todo, pelo fato de as árvores não terem o direcionamento de queda controlado. Já com relação ao Tratamento 6, a escala foi “ 3 ”, pois em todas as árvores do referido tratamento houve problema com a motosserra, que "enganchou", o que colocou em risco a segurança do operador.

Com relação aos tratamentos em que a escala foi "1", de maneira geral observou-se que, com a realização do corte de queda acima da boca de corte, houve maior segurança na operação. À medida que esse corte de queda se nivelava com a boca de corte, a segurança ficava comprometida (Tratamentos 7, 8 e 9).

\subsection{Avaliação dos defeitos}

Como pode ser observado na Figura 4a1, não ocorreu a formação de espetos em todos os tratamentos (caso dos T3, T7, T9 e T10). Nos demais tratamentos, o valor médio de volume de espetos encontrado foi de 71,84 $\mathrm{cm}^{3}$, com valor mínimo de zero e máximo de $157,50 \mathrm{~cm}^{3}$. Destaca-se que o T5, onde se aumentou o ângulo de abertura da boca de corte, foi o que apresentou maiores valores de volume de espetos (média de 122,06 cm³), visto que houve maior área para a ocorrência de espetos de maior volume.

As rachaduras de topo (Figura 4a1) foram observadas em todos os tratamentos, porém na maioria das vezes depois de transcorridas $48 \mathrm{~h}$. A média dos valores de rachadura de topo foi de $0,43 \mathrm{~cm}^{2}$, exceção feita no T9, em que o valor médio encontrado foi de $5,45 \mathrm{~cm}^{2}$. Ressalta-se que nesse tratamento foi notado imediatamente após a queda das árvores o aparecimento das rachaduras de topo, que foram progredindo durante as $48 \mathrm{~h}$.

De acordo com a Figura 4c1, nota-se que nos Tratamentos 2 e 8, em que não foram produzidos filetes de ruptura, em razão do tipo de corte, o volume de madeira remanescente na cepa foi igual a zero, haja vista que não foram produzidas tensões ao longo do tronco, o que levou ao desprendimento da madeira. Nos Tratamentos 1, 3, 4, 5, 6, 9 e 10, o valor médio encontrado de volume remanescente foi de $581,61 \mathrm{~cm}^{3}$, sendo no Tratamento 1 (técnica- padrão) obtido o menor valor $\left(460,01 \mathrm{~cm}^{3}\right)$. O T7 foi o que apresentou os maiores volumes de madeira remanescente no toco, com valores mínimo, médio e máximo de 1.820,30; 2.707,67; e 3.650, 15 $\mathrm{cm}^{3}$, respectivamente. Nesse tratamento, o filete de ruptura ficou com altura superior a $10 \mathrm{~cm}$, o que ocasionou os altos valores de madeira remanescente no toco.

\section{DISCUSSÃO}

Pelo teste de Dunnett, pôde-se comprovar a significância e variabilidade entre os dados. Através da comparação de cada tratamento com o tratamentotestemunha (T1), chegou-se aos seguintes resultados: (a) Notou-se que a profundidade da boca de corte afetou tanto a área da rachadura de topo quanto o volume de madeira remanescente nas cepas; e, ao comparar o tratamento-testemunha com os Tratamentos T3 (P $=1 / 2$ do $\mathrm{f})$ e $\mathrm{T} 4(\mathrm{P}=1 / 6$ do $\mathrm{f})$, o resultado foi significativo para rachadura de topo em ambos os tratamentos (Figura 5a), porém, ao analisar o volume de madeira remanescente, esse resultado só foi significativo na comparação entre o tratamento-testemunha e o T4 (Figura 5b). (b) Pelo teste de Dunnet, a variação do ângulo da boca de corte não produziu resultados significativos entre os Tratamentos 5 (ângulo de $65^{\circ}$ ), 6 (ângulo de $25^{\circ}$ ) e 1 . (c) Com relação à altura do corte de queda, houve significância apenas quanto ao volume de madeira remanescente, e somente o Tratamento 7 (altura $>10 \mathrm{~cm}$ ) teve significância em relação ao 1 (Figura 5c). e (d) Com relação à não execução da boca de corte, houve significância apenas para rachadura de topo; na Figura $5 \mathrm{~d}$, observa-se que os valores obtidos de rachadura de topo nos Tratamentos T2 (corte reto), T10 (corte incorreto) e no tratamento-testemunha (T1) foram significativos.

Rezende et al. (2000) atribuíram a formação de espetos nas cepas à não realização do entalhe direcional. A presença de espetos na cepa causa prejuízos à brotação, danifica a tora e leva à perda de certa quantidade de madeira.

Segundo Mellado (2007), quando a madeira seca muito rápido, exemplo típico de secagem ao ar livre de toras, gradientes de umidade excessivos são formados, o que causa contração desigual, produzindo rachaduras.

Revista Árvore, Viçosa-MG, v.36, n.3, p.511-518, 2012 


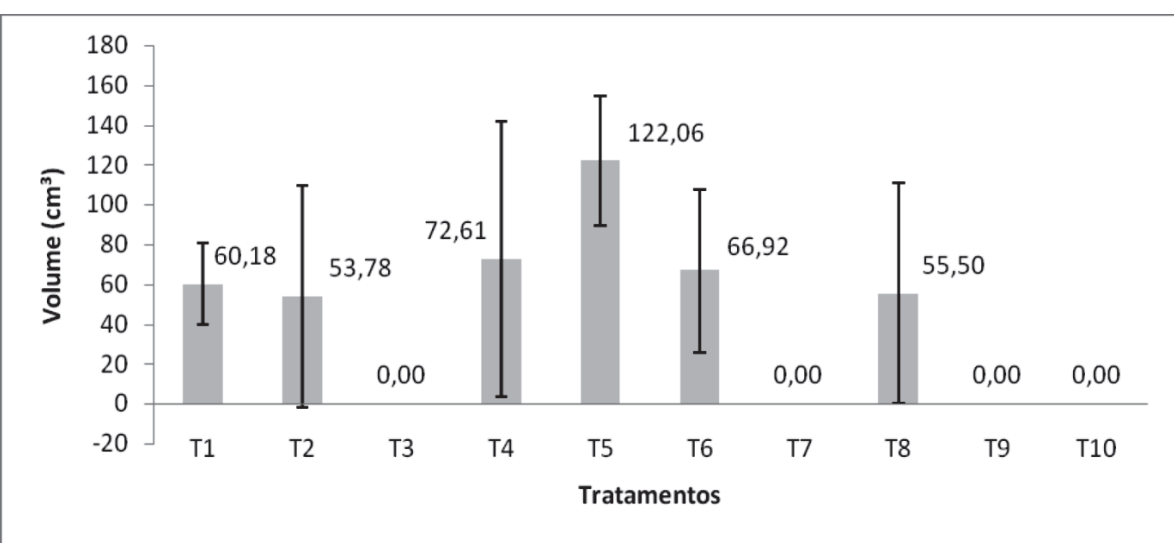

(a1)

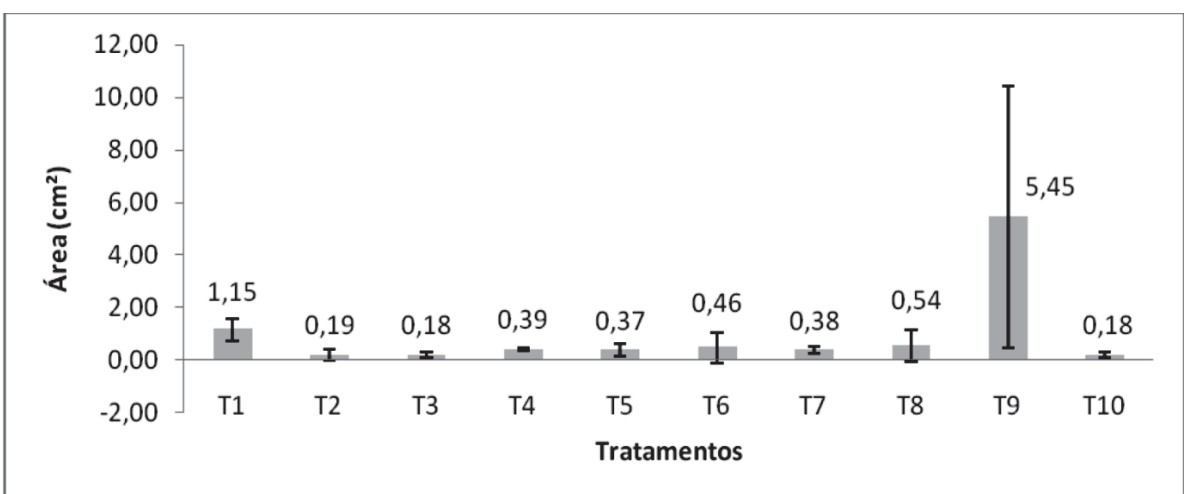

(b1)

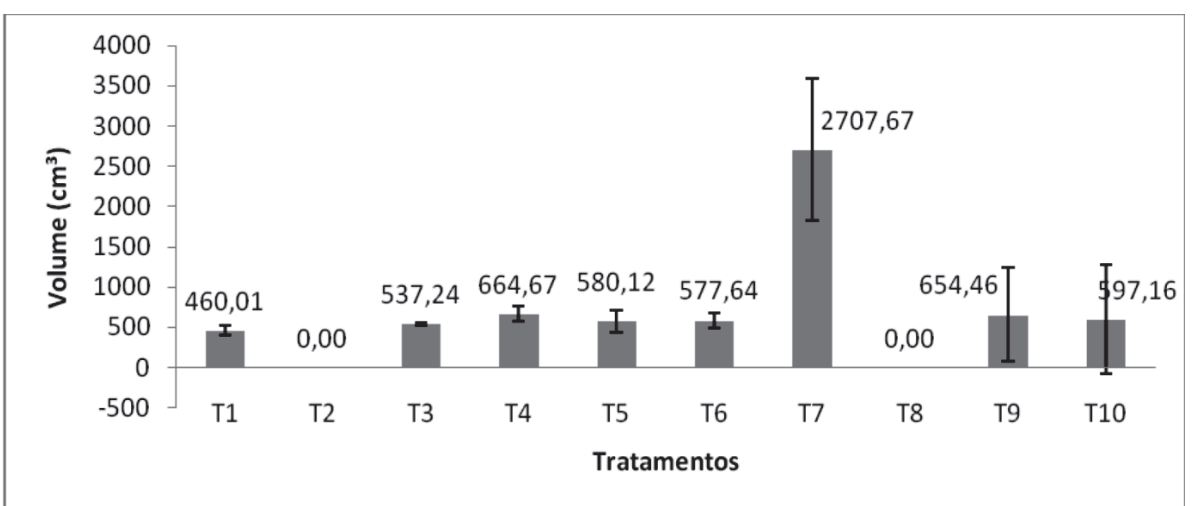

(c1)

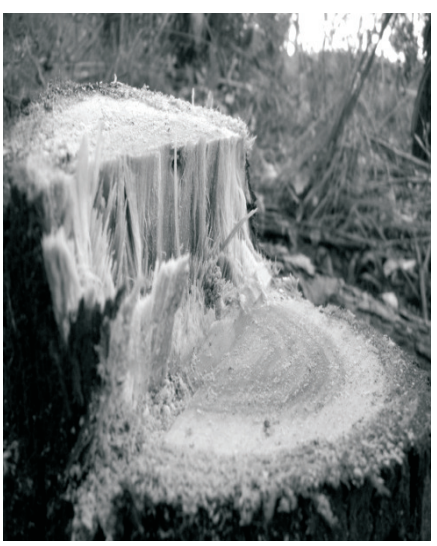

(a2)

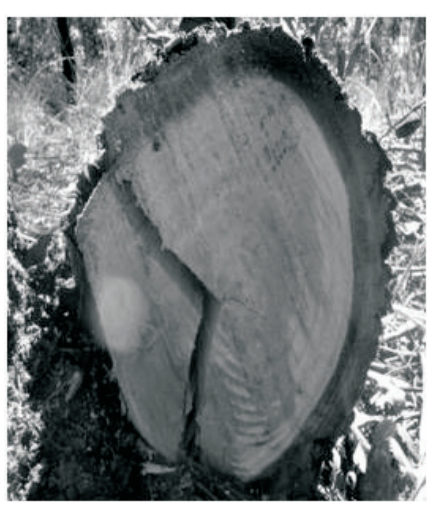

(b2)

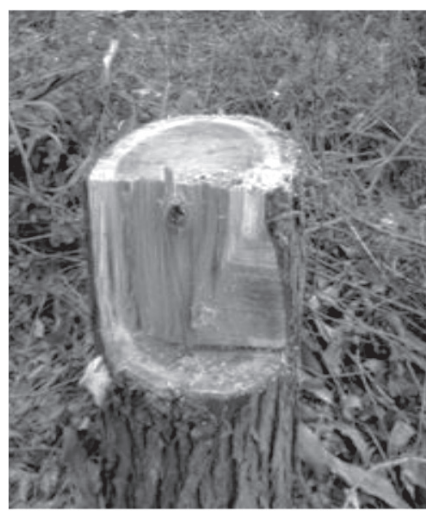

(c2)

Figura 4 - Defeitos observados: (a1) Média do volume de espeto; (a2) aspecto dos espetos no T5; (b1) Média da área de rachadura de topo; (b2) aspecto da rachadura de topo no T9; (c1) Média do volume de madeira remanescente; e (c2) aspecto de madeira remanescente no T7.

Figure 4-Observed defects: (a1)Mean value of the fork area; (a2) pattern of this variable for T5; (b1) Mean value of the end-splitting area; (b2) pattern of this variable for T9; (c1) Mean volume of the remained tree; and (c2) the pattern of this variable for the T7.

Revista Árvore, Viçosa-MG, v.36, n.3, p.511-518, 2012 


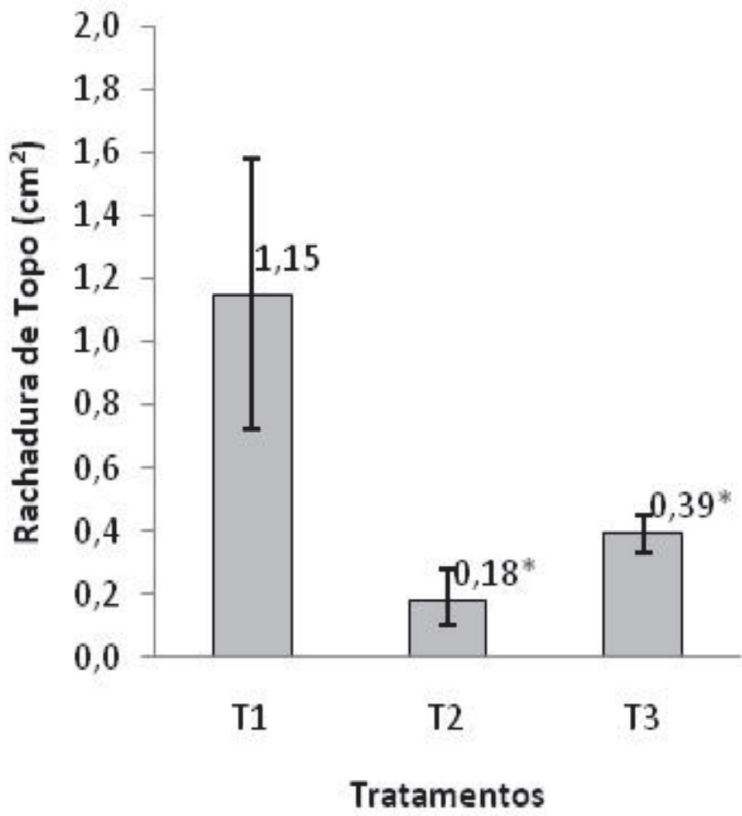

(a)

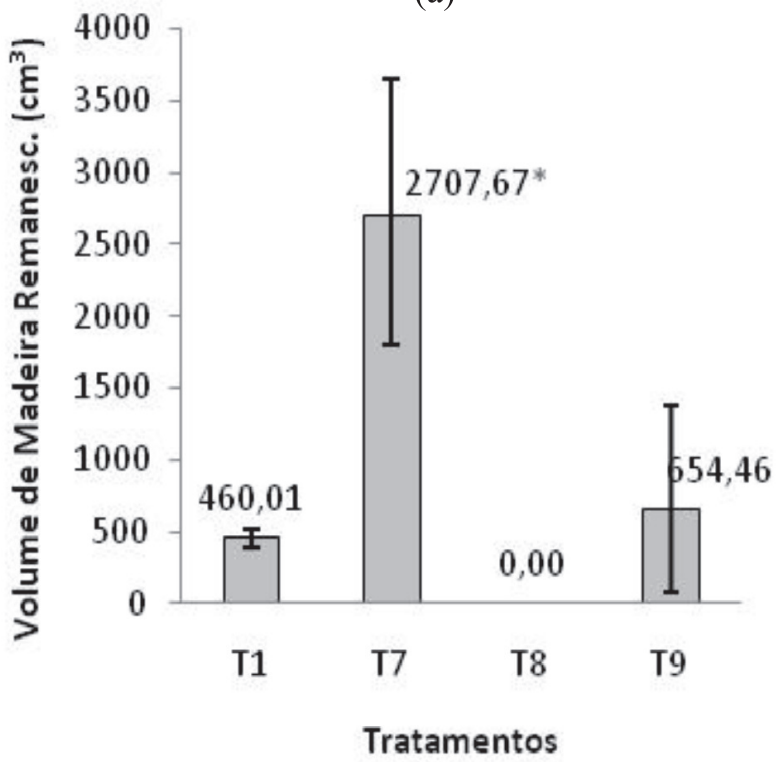

(c)

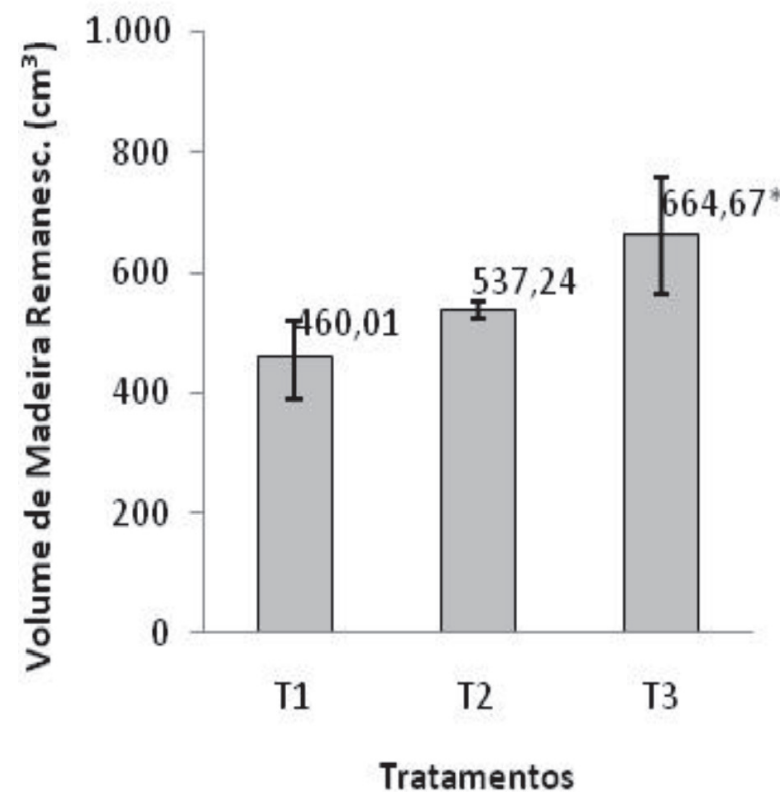

(b)

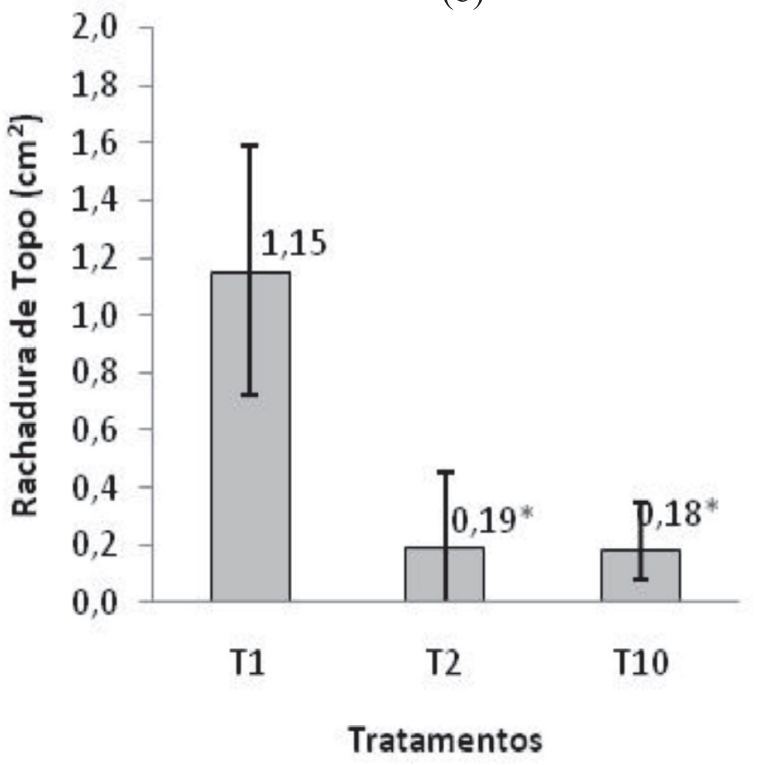

(d)

Figura 5 - Teste de Dunnet: (a) Efeito da profundidade da boca de corte na ocorrência de rachadura de topo; (b) efeito da profundidade da boca de corte no volume de madeira remanescente; (c) efeito da altura da boca de corte no volume de madeira remanescente; e (d) efeito da não execução da boca de corte corretamente na ocorrência de rachadura de topo.

Figure 5 -Dunnet test: (a) Effect of the depth fissure court in occurrence of the end-splitting; (b) effect of the depth fissure court in volume of the remained tree; (c) effect of the height fissure court in volume of the remained tree; and (d) effect of the not execution fissure court when end-splitting occurred. 


\section{CONCLUSÕES}

A segurança da operação de corte semimecanizado é afetada quando da realização de cortes fora das recomendações técnicas. Ocorreu maior número de defeitos nas situações de aumento do ângulo de corte (formação de espetos) e corte de queda abaixo (rachaduras de topo) e acima da boca de corte (madeira remanescente nas cepas). A redução da profundidade da boca de corte foi significativa tanto na área de rachadura de topo quanto no volume de madeira remanescente na cepa. O aumento da altura do corte de queda foi significativo com relação ao volume de madeira remanescente na cepa.

\section{REFERÊNCIAS}

AGUIAR, O. J. R.; JANKOWSKY, I. P.

Prevenção e controle das rachaduras de topo em tora de Eucalyptus grandis Hill ex Maiden. Piracicaba: IPEF, 1986. p.39-46.

CANTO, J. L. et al. Avaliação das condições de segurança do trabalho na colheita e transporte florestal em propriedades rurais fomentadas no Estado do Espírito Santo. Revista Árvore, v.31, n.3, p. 513-520, 2007.

JACOVINE, L. A. G., et al. Avaliação da qualidade operacional em cinco subsistemas de colheita florestal. Revista Árvore, v.29, n.3, p.391-400, 2005.

JACOBS, M. R. The growth stresses of wood stems. Bulletin Commonwealth Forestry Bureau, v.28, p.1-67, 1945.

KUBLER, H. Growth stresses in trees and related wood properties. Forest Products Abstracts, v.10, n.3, p.61-119, 1987.

LELLES, J. G.; SILVA, J. C. Problemas e soluções sobre rachaduras de topo de madeiras de Eucalyptus spp. nas fases de desdobro e secagem. Informe Agropecuário, v.18, n.186, p.62-69, 1997.

LIMA, I. L.; GARCIA, J. N.; PIEDADE, S. M. S. Rachaduras de extremidades de tora e suas implicações nas rachaduras da madeira serrada. Scientia Forestalis, n.61, p.13-24, 2002.
LIMA, I. L.; GARCIA, J. N.; NOGUEIRA, M. C. S. Influência do desbaste nas tensões de crescimento de E. grandis Hill ex-Maiden. Scientia Forestalis, n.58, p.111-125, 2000.

LOPES, E. S. et al. Operação e manutenção de motosserras: manual técnico. Viçosa, MG: Aprenda Fácil, 2001.

MACHADO, C. C.; LOPES, E. S. Análise da influência do comprimento de toras de eucalipto na produtividade e custo da colheita e transporte florestal. Cerne, v.6, n.2, p.124-129, 2000.

MELLADO, E. C. E. R. Modelo de transferência de calor e massa na secagem de madeira serrada de Pinus. 2007. 155f. Dissertação (Mestrado em Ciência Florestal) Universidade Federal do Paraná, Curitiba, 2007.

ROZAS MELlado, E. C. E. Contribuição ao desenvolvimento tecnológico para a utilização de madeira serrada de Eucalyptus grandis (Hill Ex Maiden) na geração de produtos com maior valor agregado. 1993. 133f. Dissertação (Mestrado em Ciências Florestais) - Universidade Federal do Paraná, Curitiba, 1993.

SANT'ANNA, C. M. Corte florestal. In: MACHADO, C. C. Colheita florestal. 2.ed. Viçosa, MG: Universidade Federal de Viçosa, 2008. p.55-88.

SEVERO, E. T. D.; TOMASELLI, I. Vaporização no alivio das tensões de crescimento em toras de duas procedências de Eucalyptus dunnii. Sciencia Agraria, v.1, n.1-2, p.29-32, 2000.

SKOLMEN, R. G. Heating logs to relieve growth stresses. Forest Products Journal, v.17, n.1, p.41-42, 1967.

SODERSTROM, N. Chainsaw savvy: a complete guide. New York: Morgan \& Morgan, 1982. 144p.

TRINDADE, C.; SARTÓRIO, M. L.; REZENDE, J. L. P. Controle de qualidade na exploração florestal. In: SIMPÓSIO BRASILEIRO SOBRE EXPLORAÇÃO E TRANSPORTE FLORESTAL. Anais..., Belo Horizonte: SIF/UFV, 1991. p.185-197.

WILHELMY, V.; KUBLER, H. Stresses and cheks in long ends: from relieved growth stresses. Wood Science, v.6, n.2, p.136-142, 1973. 